\title{
Prevalence, risk factors and survival in dogs with myxomatous valve degeneration
}

\author{
[Prevalência, fatores de risco e sobrevivência em cães com degeneração mixomatosa valvar] \\ J.P.E. Pascon ${ }^{1}$, G.C. Jung ${ }^{2}$, M.A. Valandro ${ }^{2}$, M.T. Oliveira ${ }^{1}$ \\ ${ }^{1}$ Universidade Federal do Pampa - Unipampa - Uruguaiana, RS \\ ${ }^{2}$ Aluno de pós-graduação - Universidade Federal do Pampa - Unipampa - Uruguaiana, RS
}

\begin{abstract}
The objective of this study was to describe the prevalence, survival time, and risk factors of myxomatous mitral valve disease (MMVD) in dogs by a retrospective observational cohort study design of a Brazilian veterinary center, admitted from 2012 to 2018 (2.585 days). Considering the 105 dog files confirmed as MMVD, the prevalence of the disease was higher in small-breed dogs with ages from 10 to 19 years (30.3\%). The survival time of the dogs at B1 stage (1.854 \pm 145 days) was higher than the B2 (1.508 \pm 209$)$ and C/D (930 \pm 209$)$. Higher risks of death (hazard ratio) were detected in the presence of syncope (2.5), pulmonary crackling (2.0), dyspnea (1.9), and higher values of vertebral heart scale (1.7), radiographic left atrium dimension (1.8), the ACVIM staging (1.7), and the duration of QRS complex (1.05). The results achieved demonstrated higher prevalence of MMVD in old small-breed dogs, associated with longer survival time in the B1 stage of the disease, and higher risk of death related to the presence of clinical signs, such as dyspnea, syncope, and pulmonary crackling, beyond some radiographic, echocardiographic, and electrocardiographic variables of heart or atrial enlargement.
\end{abstract}

Keywords: prognosis, endocardiosis, echocardiography, radiography, electrocardiography

\section{RESUMO}

O objetivo deste estudo foi descrever a prevalência, o tempo de sobrevivência e os fatores de risco da doença mixomatosa valvar mitral (DMVM) em cães, por meio de estudo de coorte observacional retrospectivo, os quais foram atendidos em um centro veterinário de 2012 a 2018 (2.585 dias). Considerando os 105 arquivos de cães com confirmação da DMVM, maior prevalência foi observada em raças pequenas com idades entre 10 e 19 anos (30,3\%). O tempo de sobrevivência de cães em estágio B1

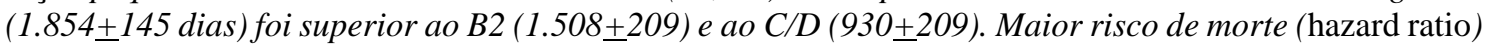
foi observado na presença de síncope (2,5), crepitação pulmonar (2,0), dispneia (1,9), bem como de valores superiores de VHS $(1,7)$, dimensão radiográfica do átrio esquerdo $(1,8)$, estadiamento ACVIM $(1,7) e$ duração do complexo QRS $(1,05)$. Os resultados obtidos demonstraram maior prevalência de DMVM em cães idosos de raças pequenas, associada a maior tempo de sobrevida no estágio B1 da doença e a maior risco de morte relacionado à presença de sinais clínicos como dispneia, síncope e crepitação pulmonar, além de algumas variáveis radiográficas, ecocardiográficas e eletrocardiográficas de cardiomegalia ou aumento atrial esquerdo.

Palavras-chave: prognóstico, endocardiose, ecocardiografia, radiografia, eletrocardiografia

\section{INTRODUCTION}

Myxomatous valve disease (MVD) is the most common cardiovascular disease in dogs, the atrioventricular valves being the most affected, in particular the mitral one (Petrus et al., 2020). Myxomatous mitral valve disease (MMVD) is the most common cause of heart failure in dogs, accounting for more than $70 \%$ of the cases reported in small-breed dogs (Borgarelli and Buchanan, 2012; Hoque et al., 2019). The prevalence of MMVD is highly associated with breed, age, body size, and sex. Males in middleaged to aged are the predominant group in most studies (Borgarelli and Buchanan, 2012). The MMVD has a diverse spectrum of severity and many dogs do not show signs of disease

Recebido em 10 de dezembro de 2020

Aceito em 18 de maio de 2021

E-mail: joaopascon@unipampa.edu.br 
progression and even do not develop congestive heart failure $(\mathrm{CHF})$, while in some cases the disease progression is evident, leading to $\mathrm{CHF}$ and even death (Kim et al., 2017; Mattin et al., 2019).

The American College of Veterinary Internal Medicine (ACVIM) published a consensus statement scheme for the disease severity, due the fact that MMVD progression varies considerably, and only a proportion of dogs show clinical signs because of the disease (Atkins et al., 2009). Alongside with the proposed staging of ACVIM, other variables obtained by ancillary exams are also being utilized as predictors from prognosis, such as the left atrium to aortic root ratio (LA/AO ratio), the vertebral heart scale (VHS) and the radiographic left atrium dimension (RLAD) (Keene et al., 2019).

Information regarding the prognostic of animals with DMV is still difficult to provide. The ACVIM classification and some variables obtained from ancillary exams have been trusted founts for the prognostic regarding disease progression and intensity; however, the prognostic referring to animals' lifespan is still under study and many times is still considered unclear. The aim of this retrospective study was to describe the prevalence and survival time, alongside with risk factors associate with MMVD in dogs that could aid in prognostication of canine valvar disease.

\section{MATERIALS AND METHODS}

A retrospective observational cohort design was used to evaluate and describe different parameters in dogs with MMVD. The study sample was obtained from medical records of dogs admitted to a Veterinary Teaching Hospital of Pampa Federal University (Brazil), from 2012 to 2018. Considering the retrospective aspect of this study without any dog interventions (only medical records), the evaluation of the ethics committee was not necessary.

All medical records contained in the MMVD as a presumptive diagnosis were investigated; however, only dogs that had a confirmed mitral valve thickening and regurgitation documented in the echocardiogram (Bagardi et al., 2020) were included in the study. In addition, the radiography and electrocardiographic recordings were mandatory for the dog to be admitted into the study. The inability to contact the owners and the concurrent diagnosis of congenital heart diseases, as well as other conditions that could interfere with the valves function, were considered exclusion criteria.

Information such as the main complaint or the first sign identified from the dogs' owners, previous medical and treatment history, information from physical examination (auscultation and heart murmur grade), clinical signs (cough, exercise intolerance, dyspnea, cyanosis, syncope, and ascites), and signalments (breed, age, sex, and weight) were collected from each individual case record. The collected radiographic variables were the vertebral heart scale (VHS) and radiographic left atrium dimension (RLAD) (Salguero et al., 2018). The variables collected from the electrocardiogram included cardiac rhythm, Pwave voltage $(\mathrm{mV})$ and duration $(\mathrm{mS})$, QRS complex voltage and duration, PQ and QT interval duration $(\mathrm{mS})$, and cardiac mean axis (Santilli et al., 2020).

The echocardiographic images and measurements of variable values were obtained according to the recommendations of the American College of Veterinary Medicine (ACVIM) (Keene et al., 2019), and included M-mode, transthoracic 2dimensional, and color Doppler imaging. The collected variables were the left ventricular internal diameter at end diastole (LVIDd) and systole (LVIDs), both normalized to body weight $\left(\right.$ normalized LVID $=\operatorname{LVID}(\mathrm{cm}) /$ weight $\left.(\mathrm{kg})^{0.294}\right)$, the septum and left ventricle free wall diameter, mitral regurgitation jet velocity and mitral regurgitation max gradient pressure, the left atrium to aortic root ratio (LA/Ao), left ventricle shortening fraction (SF) and ejection fraction $(\mathrm{EF})$, the mitral $\mathrm{E}$ wave velocity peak, $\mathrm{E}$ wave deceleration time and $\mathrm{E} / \mathrm{A}$ wave ratio $(\mathrm{E} / \mathrm{A})$, left ventricle isovolumic relaxion time, and myocardial performance index (Bagardi et al., 2020).

For survival investigation, owners were contacted by telephone. An interview was undertaken to obtain information about the animal's clinical condition. In case of death, the date and clinical signs were documented at the time of death. Whenever owners have been in emergency clinics before the dog was dead, the attending veterinarian was also contacted after obtained the owner's permission. Only the cardiovascular and 
sudden deaths were counted for the statistical analysis. For this purpose, clinical signs such as dyspnea, ascites, cyanosis, and cough were used to determine whether the death was ascribed to a cardiac situation or not. Owners that indicated sudden death were asked about any clinical signs or concurrent diseases, in order to assume a cardiovascular death. All the interviews were performed in the same period, between the months of March and June 2019.

All variables were tested for normality by Shapiro Wilk tests, and to determine the significance $(\mathrm{P}<0.05)$ of each variable, the One-Way ANOVA, Kruskal Wallis, and Chi-square tests were used, according to the normality pattern from each variable. The parameters that expressed significance in the tests were used in the survival and risk analyses. The survival analysis consisted in survival curves and mean survival times from the three groups obtained by the Kaplan-Meier method. For the survival analysis, only cardiac death was considered, minimizing dispersion and errors from the collected data. The variables that demonstrated a proportional increase in the number of deaths over time in the Kaplan-Meier analysis were considered to be in accordance with the proportional risk pattern being analyzed by the Cox regression for the determination as a risk factor (IBM SPSS ${ }^{\circledR}$ Statistics).

\section{RESULTS}

The study time comprehended the evaluation from seven years of clinical records, totalizing 2.585 days. From the first evaluation, the final number of dog files verified was 2.734 , of which 1.545 females and 1.189 males. Of the 2.734 initial dog files, 441 were from dogs attending specific cardiologic appointment. From these, 133 were indicated as a suspicion for MMVD, and 105 presented the necessary requirements to be included in this study. From the initial population (2.734) the prevalence of MMVD according to age in small breeds was $30.3 \%$ in dogs between 10 and 19 years and $23.9 \%$ in dogs between six and ten years.

Among the 105 files selected as the study sample, the mean age was $11.6 \pm 2.5$ years (range, 5-18); 62 were females (59\%), $43(41 \%)$ were males and the mean weight was $6.9 \pm 4.7 \mathrm{~kg}$ (range, 1.3 $22 \mathrm{~kg}$ ). The $105 \mathrm{dogs}$ were also classified among 13 breeds, such as Poodle $(32,30.4 \%)$, mixed breed (20, 19\%), Miniature Pinscher (13, 12.3\%), Yorkshire Terrier (13, 12.3\%), Dachshund (8, $7.6 \%)$, Fox Terrier (4, 3.8\%), Maltese (4, 3.8\%), Miniature Schnauzer (3, 2.8\%), Shih Tzu (3, $2.8 \%$ ), Pekingese $(2,1.9 \%)$, Cocker Spaniel (2, $1.9 \%)$, Beagle $(1,0.95 \%)$, and Bichon Frisé (1, $0.95 \%)$.

In accordance with the ACVIM consensus, the division of the sample resulted in $48(45.7 \%)$ dogs classified in the B1 group, $27(25.7 \%)$ in the B2, and $30(28.5 \%)$ in the C/D group. From the previously 35 variables collected from the ancillary exams and files information, 13 expressed statistical significance.

For the echocardiographic, electrocardiographic, and radiographic variables that were analyzed by the ANOVA and Kruskal Wallis tests, the ones that demonstrated signify statistical difference between the groups were the VHS $(\mathrm{P}=0.002)$, the RLAD $(\mathrm{P}=0.000)$, the LA/Ao $(\mathrm{P}=0.000)$, the normalized LVID at end systole $(\mathrm{P}=0.000)$ and diastole $(\mathrm{P}=0.002)$, and the complex $\mathrm{QRS}$ duration $(\mathrm{P}=0.002)$

As for the variables collected by the description of the dog's clinical condition during the physical examination or the description of clinical signs provided by the owner, the Chi-square test was performed. All variables demonstrated statistical significance: cough $(\mathrm{P}=0.014)$, cyanosis $(\mathrm{P}=0.017)$ dyspnea $\quad(\mathrm{P}=0.000)$, exercise intolerance $(\mathrm{P}=0.001)$, pulmonary crackling $(\mathrm{P}=0.002)$, pulmonary edema $(\mathrm{P}=0.005)$, and syncope $(\mathrm{P}=0.000)$, and the presence of the signs was mostly related to the $\mathrm{C} / \mathrm{D}$ group.

Of the 13 variables that expressed statistical difference $(\mathrm{P}<0.05)$ between the study groups and were analyzed by the Kaplan-Meyer curve for the proportional risk pattern, eight variables fit the inclusion criteria for the risk factor analysis, and seven were significant in the Cox regression. In this analysis, the group distribution was also evaluated as a risk factor, also demonstrating significance. The results for the analysis are demonstrated in Table 1. Two groups of parameters were significantly different $(\mathrm{P}<0.05)$ in the multivariable Cox analysis: the association of dyspnea, VHS, and pulmonary crackles $(\mathrm{P}<0.05)$ with a 2.0 hazard ratio (1.1-7.3), and the association of QRS duration, LA/Ao ratio, and VHS $(\mathrm{P}<0.05)$ with a 1.6 hazard ratio $(1.1-2.4)$. 
Prevalence, risk...

Table 1. Values for hazard ratio of the risk factor variables

\begin{tabular}{lcc}
\hline Variables & Hazard Ratio (CI 95\%) & P value \\
\hline ACVIM group & $1.7(1.2-2.4)$ & 0.001 \\
VHS & $1.7(1.3-2.3)$ & 0.000 \\
RLAD & $1.8(1.1-3.0)$ & 0.01 \\
QRS duration & $1.05(1.02-1.08)$ & 0.000 \\
LA/Ao ratio & $1.8(1.3-2.5)$ & 0.000 \\
Dyspnea & $1.9(1.09-3.3)$ & 0.02 \\
Syncope & $2.5(1.4-4.0)$ & 0.000 \\
Pulmonary crackling & $2.0(1.2-3.8)$ & 0.016 \\
\hline
\end{tabular}

ACVIM (American College of Veterinary Internal Medicine); VHS (Vertebral Heart Scale); RLAD (radiographic left atrium dimension); LA/Ao ratio (Left atrium to aortic root ratio); CI: confidence interval.

Out of the 105 dogs included in the survival analysis, 47 died during the observation time, and their median survival time was $1.853 \pm 108$ days. The survival time of B1 dogs (median 1.854 \pm 145 days) was higher than dogs in the B2 and C/D groups (median 1.508 \pm 209 and $930 \pm 209$ days, respectively). From the 47 dead patients, 12 $(25.5 \%)$ were in the B1 group, $15(31.9 \%)$ were in the B2 group, and $20(42.5 \%)$ were in the C/D group. Of the B1 group, $63.8 \%$ of dogs were still alive 2.585 days after the diagnosis (Fig.1).

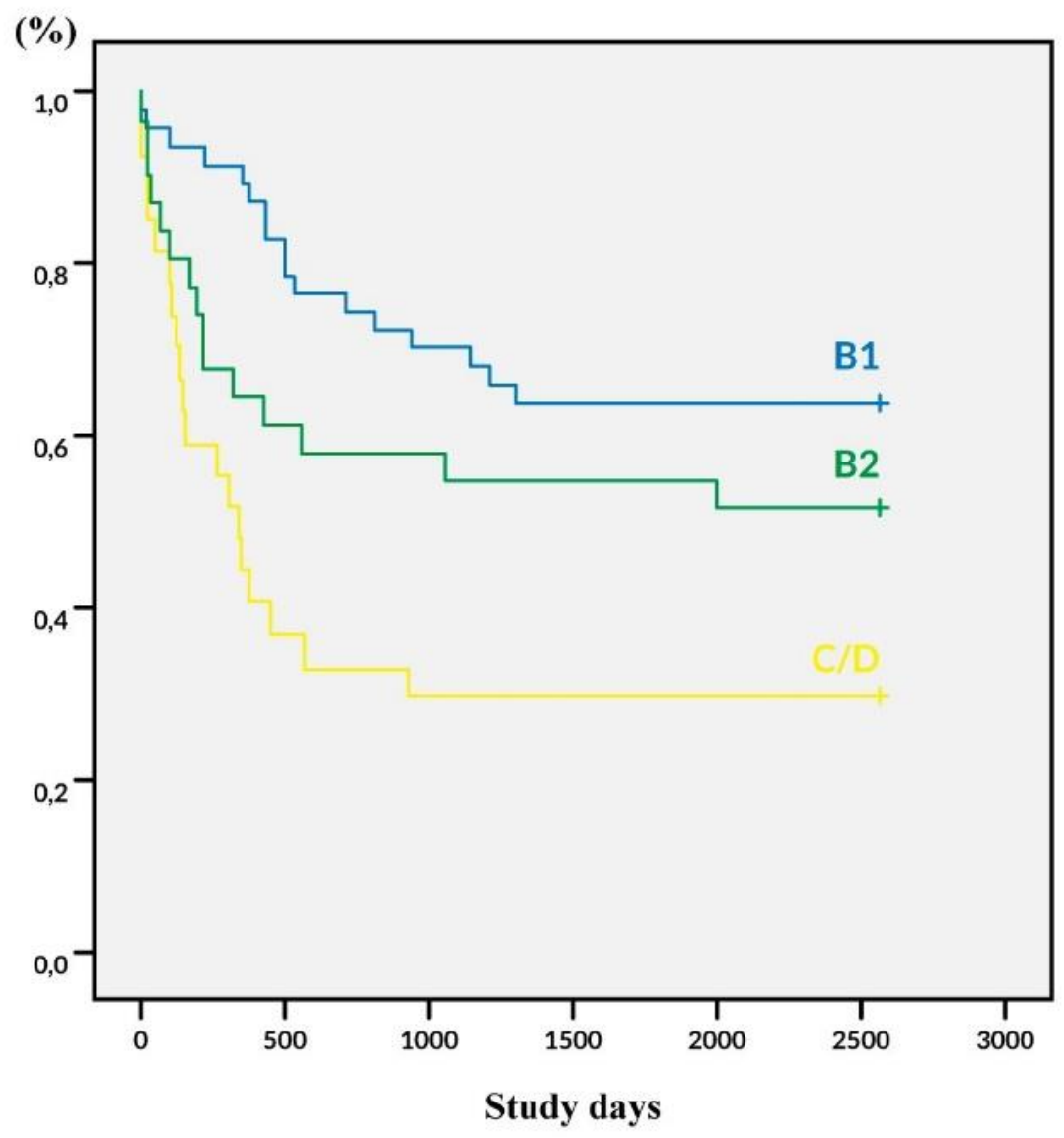

Figure 1. Survival time in 105 dogs classified by the American College of Veterinary Internal Medicine scheme. The observation time comprehended 2.585 days and $47 \mathrm{dogs}$ died in the period. The censured obits totaled 14 dogs. 


\section{DISCUSSION}

The main objective of this study was to demonstrate the prevalence, reliable risk factors, and survival in a determined population of dogs with MMDV in southern Brazil. The study sample included 105 dogs with a pre-established diagnosis of MMVD. The MMVD diagnosis demonstrated a $3.8 \%$ prevalence from the total number of clinical cases seen over the study period. However, the disease prevalence normally varies with the epidemiological characteristics, including age, sex, and breed (Borgarelli and Buchanan, 2012; Kim et al., 2017; Petrus et al., 2020). Therefore, once the sample was filtered according to characteristics from previous studies, the prevalence showed a result of $30.3 \%$, in general. The prevalence of MMVD was already described as $100 \%$ in dogs over 10 years (Borgarelli and Buchanan, 2012). Even if the results from this study have not demonstrated a similar percentile, the pattern of the disease progression according to the epidemiological characteristics is similar to other studies, demonstrating higher percentiles according to breed and age specifications (Kim et al., 2017; Mattin et al., 2019).

In this study, the number of female dogs was slightly superior to male dogs. This information is not common in MMVD studies, since the males are usually overrepresented or severely affected (Petrus et al., 2020). However, in this study, the higher number of females can be justified by the hospital caseload in the study period. From the 2.734 dogs that underwent veterinary care, 1.544 $(56.47 \%)$ were females, while $1.189(43.53 \%)$ were males. Despite that, other recent retrospective studies have shown an increase in the number of females affected by the MMVD in comparison with males (Kim et al., 2017; Mattin et al., 2019).

The evaluation of the mean age exhibited a similar behavior compared to other studies. In this study, the gap between ten and twelve years included the majority of the dogs affected. Also, the number of dogs that developed the disease in the study was increased by aging (Kim et al., 2017; Mattin et al., 2019). Age is considered a risk factor for the disease development in dogs, and many studies show a high prevalence of the MMVD associated with aging (Borgarelli and Buchanan, 2012; Keene et al., 2019). The mean weight of the dogs from this study was also a common finding among other MMVD studies, considering the disease development in small-breed dogs that unusually present weights over $15 \mathrm{~kg}$ (Kim et al., 2017; Mattin et al., 2019). Besides that, the range variation between the dogs in the study (1.3-20 $\mathrm{kg}$ ) is justified by the presence of some large breed dogs that rarely can be affected by the MMVD (Borgarelli and Buchanan, 2012; Kim et al., 2017).

The majority of breeds included in this study is also similar to the prevalence of MMVD documented in previous studies, as they are smallbreed patients, including miniatures and toys, which are the most prevalent (Borgarelli and Buchanan, 2012; Kim et al., 2017; Mattin et al., 2019). Mixed-breed dogs are the most common dog population in Brazil, and the majority of them are stray dogs. The high prevalence of mixedbreed dogs in this study is justified by the large number of these dogs attending general clinical care during the study period. In the seven years of study, 1.133 (41.4\%) mixed-breed dogs have been evaluated, and they are the largest population, followed by the miniature- and toy-breed, such as Poodles $(261 ; 9.5 \%)$ and Yorkshire terriers (147; $5.37 \%)$.

The observation of pulmonary crackling during the physical evaluation of dogs is also a considerable risk factor in this study $(\mathrm{P}=0.016)$, demonstrating twice as much risk of death for dogs with MMVD. This fact can be associated to the inefficient tissue oxygenation guiding to the aggravation of the cases. In general, the overflow is directed to the opposite way from the systolic flow, and the commonly affected mitral valve culminates in the pulmonary overflow, followed by the pulmonary edema (Borgarelli and Buchanan, 2012; Petrus et al., 2020).

The understanding of the pulmonary insufficiency as a consequence from the CHF lead to the observation of clinical signs, such as dyspnea. For dogs with impaired pulmonary function, the clinical sign of dyspnea is a way to try to optimize oxygenation, facilitating air intake, pulmonary expansion and, consequently, oxygen exchange (Morgan et al., 2020). In this study, dogs presenting dyspnea during the evaluation have at least two more chances of premature death. moreover, these chances are elevated for dogs that presented syncope (hazard ratio 2.5 ), this being a 
sign of poor prognosis, indicating oxygenation difficulty in the nervous system, and/or the presence of cardiac arrhythmias.

The pulmonary overload reflects the commonly commitment of the left side of the heart, justified by the majority of cases in the mitral valve. This overload that culminates in pulmonary congestion and edema is characterized as one of the main causes of death in dogs with MMVD (Borgarelli and Buchanan, 2012; Boswood et al., 2020). The volume overload following the events of MMVD normally ends in modifications of cardiac chambers, resulting in cardiomegaly; these structural modifications can be evaluated by techniques such as the echocardiogram and the thoracic radiographies (Keene et al., 2019). This understanding relieves the results of greater risk associated with measures that indicate left or general cardiomegaly, as the VHS, LA/Ao ratio, and RLAD.

Among the groups of the study, the C/D group has the higher mean for the VHS and LA/Ao ratio measurements, as well as it is the group with the lowest survival time. In this study the mean survival time of dogs with VHS $<10.5(2.464 \pm 81$ days) was superior to dogs with VHS >10.5 (1.374 \pm 193 days), and the hazard ratio (1.6) indicates the higher risk of death in this group. Being a measure based in two crossed measures on the heart, and compared with the dog's own vertebrae, the VHS is considered a confinable measure that indicates the general cardiomegaly based on body size (Mikawa et al., 2020). Besides being a dependable measure, subtle changes in the cardiac silhouette can be hard to identify. From this perspective, the higher the cardiomegaly, the easier it is to provide higher values for the measure, according to disease progression and severe cardiac remodeling.

The pattern demonstrated by the VHS in the C/D group was also shown when analyzing LA/Ao ratio. The mean survival time for the dogs with LA/Ao > 1.6 in this study was 1.3 times lower than that of dogs with LA/Ao <1.6. The left atrium is the chamber that commonly receives the volume overload, due to the superior incidence of the mitral valve in the MMVD. The left atrium enlargement reflects the severity of the regurgitation and the progression of the disease (Larouche-Lebel et al., 2020; Morgan et al., 2020). Being the VHS and the LA/Ao ratio measurements that indicate cardiomegaly, the results of this study are in accordance with other studies that highlight cardiomegaly as a situation associated with decreased survival in dogs (Kim et al., 2017; Salguero et al., 2018; Boswood et al., 2020).

As the measures from VHS and LA/Ao ratio, the RLAD also demonstrated significance $(\mathrm{P}<0.05)$ and high-risk factor values. The RLAD also reflect the left atrium enlargement, and it is obtained by radiographic evaluation. Similar to the LA/Ao ratio, RLAD demonstrated almost double the chance of death for dogs with values higher than 1.8 (Salguero et al., 2018). These alterations can be associated to the cardiac chamber dilatation in chronic cases of the disease, when the leak volume results in left atrium overload and enlargement, leading to severe consequences. The alterations in the VHS, LA/Ao ratio, and RLAD reflect the disease progression and the volume overflow that leads to structural changes in the cardiac chambers, the left atrium and ventricle being the structures mostly affected.

When evaluating the ventricle, the electrocardiogram might suggest chamber overload. The QRS complex provides information for the depolarization of the ventricles, the measurement of duration, suggesting the time necessary for the complete cycle during systole and diastole. In this study, the duration of QRS complex was highly associated with risk of death $(\mathrm{P}<0.001)$. The mean survival time for dogs with QRS duration $<60 \mathrm{mS}$ was $2.230 \pm 131$ days whereas for dogs with $>60 \mathrm{~ms}$ QRS duration the mean survival was $1.422 \pm 193$ days. In humans, many studies have proved the value of this variable as highly associated with the prognosis of patients with $\mathrm{CHF}$ and other cardiac diseases (Hofmann et al., 2005), but in dogs, the only study regarding this information was performed in dogs with dilated cardiomyopathy (DCM). The results are similar to those of the present study, because for dogs with DCM and QRS duration $>60 \mathrm{~ms}$, the mean survival time was much reduced when compared with the group of QRS duration $<60 \mathrm{~ms}$ (13 weeks and 25 weeks, respectively) (Pedro et al., 2011).

In this study, the association of the altered QRS duration, LA/Ao ratio, and VHS showed that the chance of death increases by 1.6 times. Previous studies demonstrate the characteristic of 
cardiomegaly as a risk factor by the exclusive evaluation of LA/Ao ratio and the VHS combined measures (Pedro et al., 2011; Mattin et al., 2019). The electrocardiographic measures are usually not demonstrated to be reliable parameters to indicate cardiomegaly (Santilli et al., 2020); however, this study showed that this measure can be useful and can increase the reliability of the alteration when combined with other variables such as the LA/Ao ratio and VHS.

As a retrospective study, some limitations could be considered. The dependency of the information in clinical records kept over the years that have been formed by different professionals, and the understanding of the significant information among the reviews is a very delicate work. In addition, insufficient information in some records or the loss of records prevented some dogs from participating in the study.

The survival analysis was based on a total of 2.585 days and the majority of dogs still alive by the end of the study belonged to either B1 or B2 stages. The dogs categorized as B1, despite having the disease, do not have clinical signs, and no treatment is recommended, therefore representing the mildest form of MMVD (Kim et al., 2017). Mitral degeneration with no compensatory alterations within the heart results in better life quality and longer survival time when compared with other stages.

Besides that, dogs in the B2 group are already experiencing consequences of the disease, including a severe cardiomegaly. This group used to have no clear indication for treatment and there was no consensus regarding therapy in these dogs. However, in 2016 the EPIC study demonstrated the benefits of treating B2 dogs with Pimobendan. In these dogs, the median time to the onset of $\mathrm{CHF}$ or cardiac-related death was prolonged by approximately 15 months, while the risk of a dog experiencing this event was reduced by approximately one-third (Boswood et al., 2016).

In this study, the survival curves for the B1 and B2 groups were slightly similar, and $63 \%$ of the dogs from the B1 group were still alive by the end of the study, while $51 \%$ of the dogs of the B2 group were also alive by the end of 2.585 days. The difference between the survival days estimative was smaller between the B1 and B2 groups (346 days) when compared with the difference between the B1 and C/D groups (924 days). The similarity in the curves and the survival days estimative can be explained by the number of dogs, according to the attendance year. In this study, the majority of the dogs were first evaluated between 2016 and 2018; in view of this information, many dogs in the study are receiving the Pimobendan therapy.

In addition, some dogs that were classified in the B2 stage after the EPIC study release were also indicated to use this medication during reevaluations. For the B2 group, $51 \%$ of the dogs were still alive by the end of the study, besides $29 \%$ from the C/D group. As indicated by other studies, the use of Pimobendan prolonged survival time of the B2 dogs in this study (Boswood et al., 2016; Keene et al., 2019). In this study, the dogs in the B1 group lived approximately 11 months more than the dogs in the B2 group, and 31 months more than the dogs in the $\mathrm{C} / \mathrm{D}$ group. In addition, dogs from B2 group lived approximately 19 months more than those in the C/D group.

The dogs in the C/D group, demonstrated the lowest survival curve. The onset of CHF is a welldetermined risk factor in dogs with MMVD, and this means a poor prognosis these animals (Keene et al., 2019; Mattin et al., 2019; Boswood et al., 2020). All dogs in this group have already experienced $\mathrm{CHF}$. This fact highlights heart health and its capacity of maintaining its normal functions. At this moment the compensatory mechanisms are activated, and other changes start to become deleterious for the organism. The necessity of therapy management in these cases include potent diuretic administrations that could also overburden the kidneys (Keene et al., 2019; Mattin et al., 2019). In general, the incapacity of the heart to maintain its role normally culminates in the dog's death by the progressive disease, resulting in the development of pulmonary edema, or the choice of euthanasia (Keene et al., 2019; Boswood et al., 2020).

Heart incapacity, the overburden of other organic systems, and the necessity to combine medications due to disease progression, when evaluated according to the division of the sample by ACVIM staging, shows that the survival analysis for this criterion follows the proportional risk pattern. From this view, the ACVIM staging was identified as a very significant risk factor $(\mathrm{P}<0.001)$. According to different studies, in some 
cases the disease progression can be very aggressive, and this is associated with a poor quality of life and low survival time (Keene et al., 2019; Boswood et al., 2020; Morgan et al., 2020). To the dogs that are in an advanced stage, according to the ACVIM consensus, the hazard ratio demonstrated approximately twice the chance of premature death. This result can be mainly associated with heart insufficiency, as well as the consequent volume overload (Morgan et al., 2020).

\section{CONCLUSION}

In conclusion, the higher prevalence of MMVD in the study was detected in small breed dogs between 10 and 19 years, with higher risk of death in the presence of clinical signs, such as dyspnea, syncope, pulmonary crackling, or radiographic (VHS and RLAD), echocardiographic (LA/Ao), and electrocardiographic (QRS duration) evidences of heart or left atrium enlargement, which corresponds to the ACVIM staging of the disease and reflects in longer life time in the B1 stage compared with B2 or C/D stage.

\section{ACKNOWLEDGMENTS}

This study was financed in part by the Coordenação de Aperfeiçoamento de Pessoal de Nível Superior - Brazil (CAPES) Finance Code 001. To the Veterinary Cardiology Service in the Federal University of Pampa Veterinary Hospital (HUVet UNIPAMPA) and Federal University of Pampa.

\section{REFERENCES}

ATKINS, C.; BONAGURA, J.; ETTINGER, S. et al. Guidelines for the diagnosis and treatment of canine chronic valvar heart disease. J. Vet. Intern. Med., v.23, p.1142-1150, 2009.

BAGARDI, M.; BIONDA, A.; LOCATELLI, C. et al. Echocardiographic evaluation of the mitral valve in Cavalier King Charles Spaniels. Animals, v.9, p.1454-1469, 2020.

BORGARELLI, M.; BUCHANAN, J.W. Historical review, epidemiology and natural history of degenerative mitral valve disease. $J$. Vet. Cardiol., v.14, p.93-101, 2012.
BOSWOOD, A.; GORDON, S.G.; HÄGGSTRÖM, J. et al. Temporal changes in clinical and radiographic variables in dogs with preclinical myxomatous valve disease: the EPIC study. J. Vet. Intern. Med., v.34, p.1108-1118, 2020 .

BOSWOOD, A.; HAGGSTROM, S.G.; GORDON, G. et al. Effect of pimobendan in dogs with preclinical myxomatous mitral valve disease and cardiomegaly: the EPIC study- a randomized clinical trial. J. Vet. Intern. Med., v.30, p.17651779, 2016.

HOFMANN, M.; BAUER, R.; HANDROCK, R. et al. Prognostic value of the QRS duration in patients with heart failure: a subgroup analysis from 24 centers of Val-HeFT. J. Card. Fail., v.7, p.523-528, 2005.

HOQUE, M.; SAXENA, A.C.; GUGJOO, M.B. et al. Cardiac diseases in dogs. Indian J. Anim. Health, v.58, p.1-20, 2019.

KEENE, B.W.; ATKINS, C.E.; BONAGURA, J.D. et al. ACVIM consensus guidelines for the diagnosis and treatment of mixomatous mitral valve disease in dogs. J. Vet. Intern. Med., v.33, p.1-14, 2019.

KIM, T.H.; HAN, S.M.; SONG, W.J. et al. Retrospective study of degenerative mitral valve disease in small-breed dogs: survival and prognostic variables. J. Vet. Sci., v.18, p.369-376, 2017.

LAROUCHE-LEBEL, E.; LOUGHRAN, K.A.; OYAMA, M.A. Echocardiographic indices and severity of mitral regurgitation is dogs with preclinical degenerative mitral valve disease. $J$. Vet. Intern. Med., v.33, p.484-498, 2019.

MATTIN, M.J.; BOSWOOD, A.; CHURCH, D.B. et al. Prognostic factors in dogs with presumed degenerative mitral valve disease attending primary-care veterinary practices in the United Kingdom. J. Vet. Intern. Med., v.33, p.432-444, 2019.

MIKAWA, S.; NAGAKAWA, M.; OGI, H. et al. Use of vertebral left atrial size for staging of dogs with myxomatous valve disease. J. Vet. Cardiol., v.30, p.92-99, 2020. 
MORGAN, K.R.S.; MONTEITH, G.; RAHEB, S. et al. Echocardiographic parameters for the assessment of congestive heart failure in dogs with myxomatous valve disease and moderate to severe mitral valve disease and moderate to severe mitral valve regurgitation. Vet. J., v.263, p.1-6, 2020.

PEDRO, B.M.; ALVES, J.V.; CRIPPS, P.J. et al. Association of QRS duration and survival in dogs with dilated cardiomyopathy: a retrospective study of 266 clinical cases. J. Vet. Cardiol., v.13, p.243-249, 2011.
PETRUS, L.C.; GIMENES, A.M.; MANTOVANI, M.M. Degeneração mixomatosa valvar. In: LARSSON, M.H.M.A. (Ed.). Tratado de cardiologia de cães e gatos. São Caetano do Sul: Interbook, 2020. p.155-170.

SALGUERO, X.S.; PRANDI, D.; LLABRÉSDIAZ, F. et al. A radiographic measurement of left atrial size in dogs. Ir. Vet. J., v.71, p.25-32, 2018.

SANTILLI, R.; MOÏSE, N.S.; PARIAUT, R. (Eds.). Eletrocardiografia de cães e gatos, diagnóstico de arritmias. 2.ed. São Paulo: Medvep, 2020. 358p. 